$6^{\circ}$, and a separate scale is used for every different value of the index $h$. For structure factor summations, the numbers on the scales represent successive values of the index $h$, and a separate scale is used for every different value of the co-ordinate $\theta_{1}$, the number of scales required being equal to the number of atoms in the asymmetric unit.

In practice, the calculation of a number of structure factors can be made with the same facility as the calculation of the electron density at the same number of points on a projection of the structure. The first factor, $A \cos h \theta_{1}$, is evaluated for a number of values of $h$, and these results are used as coefficients for the final summations. The method is particularly useful for structures containing large numbers of atoms of rather similar scattering power, such as occur in organic compounds, where sufficient accuracy can be obtained by using the same atomic $f$-curve for all the atoms, but weighting the coefficients in proportion to the atomic numbers.

As a further supplement to my previous paper ${ }^{3}$, I should like to point out that the Fourier synthesis of non-centrosymmetrical projections can be carried out very easily either by my method or that of Beevers and Lipson if the arbitrary phase constant, $\alpha$, is first combined with the measured $F$ values. The series

$$
\Sigma \Sigma F \cos \left(h \theta_{1}+k \theta_{2}-\alpha\right)
$$

may be written

$\Sigma \Sigma\left[\left(F^{\prime} \cos h \theta_{1}+F^{\prime \prime} \sin h \theta_{1}\right) \cos k \theta_{2}-\left(F^{\prime} \sin \right.\right.$ $\left.\left.h \theta_{1}-F^{\prime \prime} \cos h \theta_{1}\right) \sin k \theta_{2}\right]$

where $F^{\prime \prime}=F \cos \alpha$ and $F^{\prime \prime}=F \sin \alpha$.

Examples of such projections are given in a recent paper on the structure of resorcinol ${ }^{4}$. Further simplification of the formulæ often follows from relations between the phase constants in the different quadrants of the reciprocal lattice. These relations are summarized for the different space groups in a recent book by Dr. Lonsdale ${ }^{5}$.

J. Monteath Robertson.

Davy Faraday Laboratory,

Royal Institution,

London, W.1.

Sept. 22.

1 NATURE, 137, 825 (1936).

2 NATURE, 138, 362 (1936).

- J. M. Robertson, Proc. Rou. Soc., A, in the press.

" "Structure Factor Tables", by K. Lonsdale (G. Bell and Sons).

\section{Self-Interaction of Neutrons and Protons}

IT is known that, starting with Fermi's expression for the coupling between a heavy nuclear particle and the field of electrons and neutrinos, we get in the second approximation the law for interaction between proton and neutron ${ }^{1}$. This law :

$$
\sim \frac{g^{2}}{h c} \frac{(h / m c)^{28}}{r^{5+28}}
$$

( $g$ is Fermi's constant, the best choice for $s$ is 3 ) gives a divergent result at $r \rightarrow 0$, which shows the impossibility of constructing a point model of a heavy particle, just as the classical and quantum electrodynamics both lead to infinite self-interaction of a point charged particle on Coulomb's formula. The only reasonable method so far proposed to remove this difficulty of classical electrodynamics is the nonlinear generalization of Maxwell's equations developed by Mie and Born ${ }^{2}$, who introduced a new characteristic length (the analogue of the radius of the electron).

Fermi's theory also introduces a universal length $r_{0}=\sqrt{g / h c} \sim 10^{-16} \mathrm{~cm} .$, or better $r_{0}=\sqrt[\delta+2]{(h / m c)^{\delta} g / h c}$ $\sim 10^{-13} \mathrm{~cm}$. with $s=3$, say, which fact changes the whole situation of the interaction problem, as well as that of the theory of scattering ${ }^{3}$. Let us compute the interaction between heavy particles transferred not only by a single pair, but by $2,3 \ldots n$ pairs of light particles, electrons and neutrinos. In electro. dynamies this would correspond to the transfer of energy by $2,3 \ldots n$ photons, afterwards emitted and absorbed by two charged particles. Putting $\psi=\psi_{0}+\psi_{1}+\ldots \psi_{n}$ for the wave function of two heavy particles, we get for the interaction, as far as the $2 n$th approximation :

$$
V=-\frac{g^{2}}{h c}\left(\frac{h}{m c}\right)^{28} \frac{1}{r^{28+5}} \sum_{n=0}^{\infty}(-1)^{8} \alpha_{n}\left(\frac{r_{0}}{r}\right)^{2 n(2+8)}
$$

where $\alpha_{n}$ are numerical coefficients of the order unity. At great distances $\left(r \gg r_{0}\right)$ we obtain, of course, the previous law, but at small distances $r<r_{0}$ the successive members of the series are of the same order of magnitude, which corresponds physically to the intensive interaction transfer not only by one pair but also by many pairs of light particles, or as we may say, by showers of particles.

Though at $r \rightarrow 0$ each member of the series tends to infinity, the whole sum can be finite, which is best illustrated by the example of the following expression, the expansion of which is quite similar to our series, differing from it possibly only in the values of numerical coefficients :

$$
V=\frac{g^{2}}{h c}\left(\frac{h}{m c}\right)^{2 s} \frac{1}{\left(r^{28+4}+r_{0}^{28+4}\right)^{1+1} \overline{1(28+4)}}
$$

As the exact values of the coefficients are at present not of great importance owing to the preliminary character of the whole of Fermi's theory, it is very attractive to admit this law as the general form of interaction, giving the required finite proper energy of a heavy particle $M c^{2}$ at $r \rightarrow 0$, just as the potential of Born's non-linear theory leads to the finite value of $m c^{2}$ for the electron. We find in this manner that Fermi's $r_{0}$ plays the role of the radius of a heavy particle. We see that by taking into account the shower-like character of energy transfer, realized by many particles, or photons, we are led immediately to the non-linear law of interaction.

D. Iwanenko.

A. Sokolow.

Siberian Physical Technical Institute,

Tomsk.

Sept. 13.

${ }^{1}$ D. Iwanenko and A. Sokolow, Verhandl. d. Sibirisch, Phys.-Techn. ${ }^{1}$ D. Iwanenko and A. Sokolow, Verhandl. d. Sibirisch, Phy8.-Techn.
Instituts, (Russ.), 4, 67 (1936). NATURE, 138, 246 (1936). Z. Phys., Instituts, (Russ.)

${ }^{2}$ M. Born, Proc. Roy. Soc., A, 143, 410 (1934).

' W. Heisenberg, Z. Phys., 101, 119 (1936).

\section{A New Kind of Ring Phenomenon in Sputtered Metallic Films}

AN interesting ring phenomenon which is expected to throw light on the mechanism of condensation of sputtered particles was noticed while working on cathodic sputtering. Details will be published elsewhere.

It was noticed that if a small bead of plasticine or a small drop of oleic acid is placed on a specially 